\title{
Evaluación del comportamiento mecánico de asfalto natural a partir de muestras a temperatura ambiente provenientes de Caquetá, Colombia
}

\section{Assessment of mechanical response of natural asphalt using ambient temperature samples from Caquetá, Colombia}

Johanna Carolina Ruíz-Acero'
Oscar Javier Reyes-Ortiz
Luis Ángel Moreno-Anselmi

Recibido: diciembre 01 de 2014

Aceptado: noviembre 03 de 2015

\begin{abstract}
Resumen
El objetivo de esta investigación fue evaluar el comportamiento mecánico del asfalto natural por medio de la fabricación de muestras a temperatura ambiente. En primera instancia se realizó la caracterización del material con el ensayo de extracción cuantitativa del asfalto y así conocer el contenido de asfalto presente en el material; luego se procedió a fabricar muestras con la asfaltita pura en el compactador giratorio y por último se ejecutó sobre estas el ensayo de Resistencia a Tracción Indirecta, RTI, en estado seco y en estado húmedo, para así conocer la viabilidad de su uso en vías terciarias, sin tener que someterla a altas temperaturas de fabricación y mezclado. Como resultado principal se evidencia que las probetas fabricadas a temperatura ambiente presentan resistencias muy bajas, por lo cual es necesario probar con la adición de agregados pétreos a la misma temperatura y emulsiones asfálticas.
\end{abstract}

Palabras clave: asfalto natural, Resistencia a Tracción Indirecta, pavimentos flexibles.

\begin{abstract}
The aim of this research was to evaluate the mechanical behavior of natural asphalt by making samples at ambient temperature. It was made a material characterization testing, using quantitative extraction of bitumen to know the asphalt content in the material. Then, it was proceeded to fabricate samples with pure natural asphalt in the gyratory compactor, and finally, samples were assayed by indirect tensile strength test in dry and wet conditions to know the viability of its use in tertiary roads without being subjected to high temperatures in manufacturing and mixing. The main result is evidence that the specimens fabricated at room temperature have very low resistance, so it is necessary to test the addition of stone aggregates at the same temperature and asphalt emulsions.
\end{abstract}

Keywords: natural asphalt, indirect tensile strength test, flexible pavements. 


\section{Introducción}

En Colombia, para la construcción de vías tanto principales como secundarias y terciarias, es frecuente el uso de pavimentos flexibles, compuestos generalmente por una capa de rodadura y una base asfáltica que contiene la mezcla de agregados, asfalto y si es necesario aditivos, sometidos a temperaturas entre los 150 y 180 grados centígrados para fabricación. El asfalto en mención, proviene de la extracción y procesamiento del petróleo en los diferentes pozos existentes en el país, dando lugar a la obtención del asfalto como uno de los residuos generados por el crudo. No obstante, su forma de producción genera altos niveles de contaminación ambiental y emisión de gases tóxicos, lo cual a nivel mundial ha creado una conciencia en la búsqueda de disminuir dicha contaminación y emisión de gases del efecto invernadero (Spielmann, 2000; Ruíz, Reyes, \& Moreno, 2013).

Por lo anterior, distintas investigaciones buscan nuevos productos o medios que reduzcan en gran medida las inconformidades medioambientales, teniendo como alternativa el asfalto natural, también conocido como asfaltitas. El reemplazo de asfalto por asfalto natural, podría servir en algunas zonas del país, especialmente en aquellas donde la extracción de asfalto natural es abundante y los recursos distribuidos por el estado son escasos, generando mayor accesibilidad de las poblaciones a los centros urbanos, brindando un desarrollo económico óptimo y de igual manera, fuentes de trabajo en el sitio (Vázquez, 2010).

El asfalto natural se define como una sustancia sólida combinada con materiales pétreos, material arenoso y fino, de distintos tamaños y formas, que presenta una consistencia viscosa y su punto de fusión empieza desde los 110 grados centígrados; tiene su origen de varios yacimientos de rocas asfálticas, por lo general calizas, areniscas y gravas conglomeradas impregnadas de asfalto natural, las cuales favorecen la fabricación de mezclas asfálticas porque al combinarse con agregados permite ligar de manera óptima la asfaltita con el agregado permitiendo ser empleadas en las regiones como componentes de pavimentos de carreteras (Berry \& Reid, 1993).

A nivel internacional diferentes academias han desarrollado investigaciones que abarcan la asfaltita; como muestra de esto, en Estados unidos, el Strategic Highway Research Program, SHRP, con el fin de mejorar el grado de rendimiento de las especificaciones de las carpetas de asfalto usaron un tipo de asfalto natural denominado la gilsonita para buscar un rango de temperatura en el cual se debería aplicar la mezcla para no afectar su rendimiento. Este asfalto fue empleado como aditivo estabilizador, que buscaba mejorar el rendimiento de las bases asfálticas aportando flexibilidad a bajas temperaturas. Su función como agente modificador del asfalto es semejante a la implementación de fibras de caucho, que brinda las mismas propiedades pero no tiene necesidad de ser procesada para poder utilizarse (Osborn, 2002).

Adicionalmente en Brasil, se realizó un estudio en el cual se fabricaron mezclas asfálticas modificadas con asfaltita dando como conclusión un rendimiento mayor, y un aumento en las propiedades mecánicas y dinámicas de la mezcla en comparación con el asfalto usado comúnmente (extraído de pozos petroleros). La mezcla fue conformada por agregado de basalto, arena y cal hidratada que al combinarse con el asfalto natural otorgó una mayor resistencia a la deformación permanente en comparación con el uso de asfaltos convencionales. Así mismo, este estudio evidenció que las propiedades tales como la susceptibilidad térmi$\mathrm{ca}$, la resistencia a la fatiga, resistencia a los daños por humedad, módulo de elasticidad y resistencia a la tracción indirecta aumentan, pero también con una serie de desventajas tales como la afectación que tiene el clima sobre las muestras, las altas temperaturas y fuertes lluvias (Rohde, Pereira, Pérez, \& Vitorello, 2008).

A nivel nacional, en Bogotá la Universidad de Los Andes realizó un estudio para mostrar la tecnificación del uso de asfaltitas de la mina de San Pedro, 
presentando como resultados una extracción de asfalto entre el 2 y $2.5 \%$, un índice de lajas del 9\%, índice de agujas de $12 \%$, desgaste en la máquina de los Ángeles de $29.3 \%$, equivalente de arena de $91.8 \%$ y tamaño máximo nominal de 1"; parámetros que logran inferir una humedad adicional presente en el asfalto natural y que por tanto hace que mejore la estabilidad a corto plazo; por los contenidos de volátiles la densidad se ve afectada en gran medida; sin embargo, se ajustan a las especificaciones estipuladas para carpetas asfálticas (Rohde et al., 2008). Otro estudio con resultados similares a los anteriores, concluyó que las asfaltitas constituyen una alternativa para la pavimentación y mantenimiento vial, afirmación sustentada en la bondad del material mostrada a través de los satisfactorios resultados de los diferentes ensayos de laboratorio: Módulo Resiliente y Diseño Marshall; el limitante de este producto natural radica en la variación tanto de la granulometría, como el asfalto residual, factores que son extremadamente relevantes en la medida en que se omitan los controles necesarios antes y durante la construcción de la estructura (Barbosa, 2003).

Rondón y Reyes (2012), evaluaron en el laboratorio mezclas asfálticas MDC-2, con asfalto $60-70$ y 80-100, modificadas con asfaltita provenientes del Cesar, Colombia, en porcentajes de 0,5, 1,5, 2,5 y $3,5 \%$. Ejecutaron ensayos de resistencia mecánica bajo carga monotónica, módulo resiliente y resistencia a deformación permanente, concluyendo un incremento en la resistencia mecánica de 3.84 veces cuando se adiciona $0,5 \%$ de asfaltita con asfalto $80-100$ y un incremento de 1.89 veces al adicionar $2.5 \%$ de asfaltita para el asfalto tipo 60/70. En el estudio, la rigidez y resistencia a la deformación permanente, incrementa para el asfalto 80-
100 entre $51-113 \%$ y para el asfalto $60-70$ entre 35 $64 \%$, con porcentajes de asfaltita entre 0.5 y $3.5 \%$.

Los estudios anteriores muestran el potencial del asfalto natural como material para la pavimentación de vías terciarias; esto es muy importante en departamentos como Caquetá, Colombia, que poseen minas de asfalto natural, y donde de los 4.000 $\mathrm{km}$ de vías terciarias, solo el $0,5 \%$ se encuentran pavimentadas.

La presente investigación pretende dar a conocer el comportamiento mecánico de muestras fabricadas a partir de la asfaltita pura, extraída de Caquetá, sin ser sometida a cambios de temperatura, con el fin de conocer su viabilidad ante la puesta en obra sobre vías terciarias. El asfalto natural se sometió a pruebas de Resistencia a Tracción Indirecta, RTI, a temperatura ambiente, llegando a la conclusión que las muestras se deben mejorar adicionando al asfalto natural material granular y emulsión asfáltica.

\section{Materiales y métodos}

\subsection{Materiales}

El asfalto natural se extrajo de la mina ubicada a $30 \mathrm{~km}$ iniciando en el PR 54 del municipio de San Vicente del Caguán, Caquetá, saliendo hacia Neiva por la vía Minas Blancas-Santo Domingo-Balsillas, Neiva, en el PR 78. Como se observa en la tabla 1, se tomó la norma I.N.V.E-732-07 para realizar el ensayo de extracción cuantitativa del asfalto en mezclas en caliente para pavimentos, para calcular posteriormente el porcentaje de asfalto contenido en las asfaltitas, siendo en promedio de 13,10\%. 


\begin{tabular}{ll}
\hline \multicolumn{1}{c}{ Ítem } & \multicolumn{1}{c}{ Peso (g) } \\
\hline Peso recipiente & 116,9 \\
Muestra inicial + recipiente & 1116,9 \\
Muestra sin centrifugar & 1000,0 \\
Después de lavado con gasolina & 989,4 \\
Después de lavado con gasolina sin recipiente & 872,5 \\
Después meterla al horno + recipiente & 983,4 \\
Después meterla al horno & 866,5 \\
Después de lavado + recipiente & 954,3 \\
Después de lavado & 837,4 \\
Material pasa 200 & 29,1 \\
Filtro antes del centrifugado & 14,8 \\
Filtro después del centrifugado & 17,3 \\
Material en el filtro & 2,5 \\
Peso total & 869,0 \\
\% Asfalto & 13,1 \\
\hline
\end{tabular}

Tabla 1. Pesos tomados para calcular el contenido de asfalto en la asfaltita.

\subsection{Metodología}

La metodología de la presente investigación se muestra en la figura 1. La primera etapa inicia con la caracterización del material, por medio del ensayo de extracción cuantitativa del asfalto en mezclas en caliente para pavimentos, I.N.V.E-732-07, y de esta manera conocer el contenido de asfalto en el asfalto natural (INVÍAS, 1997). En seguida se fabricaron muestras de asfalto natural en el compactador giratorio, según especificaciones SUPERPAVE; dicha fabricación se efectuó sin calentar la asfaltita, esto quiere decir que se realizó el mezclado del material a temperatura ambiente. Luego se procedió a realizar el ensayo de Resistencia a Tracción Indirecta para muestras en estado seco y en estado húmedo. Para finalizar fue posible establecer el uso de asfaltitas teniendo en cuenta la fabricación de muestras cilíndricas a temperatura ambiente.

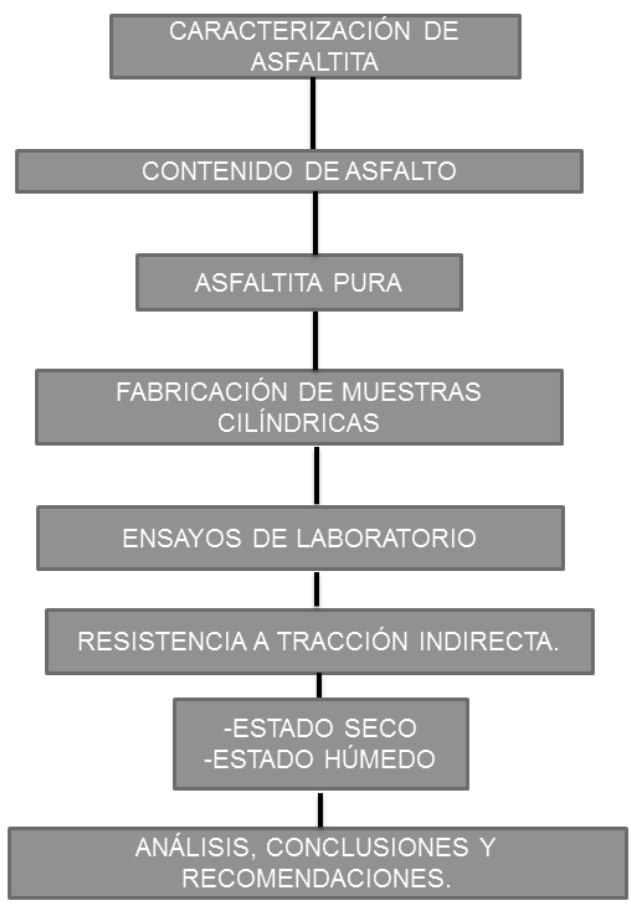

Figura 1. Metodología de la investigación. 


\section{Resultados y discusión}

Teniendo en cuenta que el asfalto natural presenta una heterogeneidad considerable, se dividió el material en cuatro zonas de la misma cantera, así: Zona 1, Zona 2, Zona 3 y Zona 4, para así presentar los resultados de la asfaltita proveniente de $\mathrm{Ca}$ quetá, después de ser falladas a Resistencia a Tracción Indirecta en estado seco y en estado húmedo.
En la figura 2 se observa que de las tres muestras significativas reportadas en esta investigación, ensayadas en estado seco, la muestra número 1 presentó mayor resistencia que las otras dos, pasando los $6 \mathrm{Kg}$ de resistencia. En general, el comportamiento de las tres muestras es similar antes y después de la falla. La muestra número 1 presentó mayor desplazamiento, llegando a 12,00 mm.

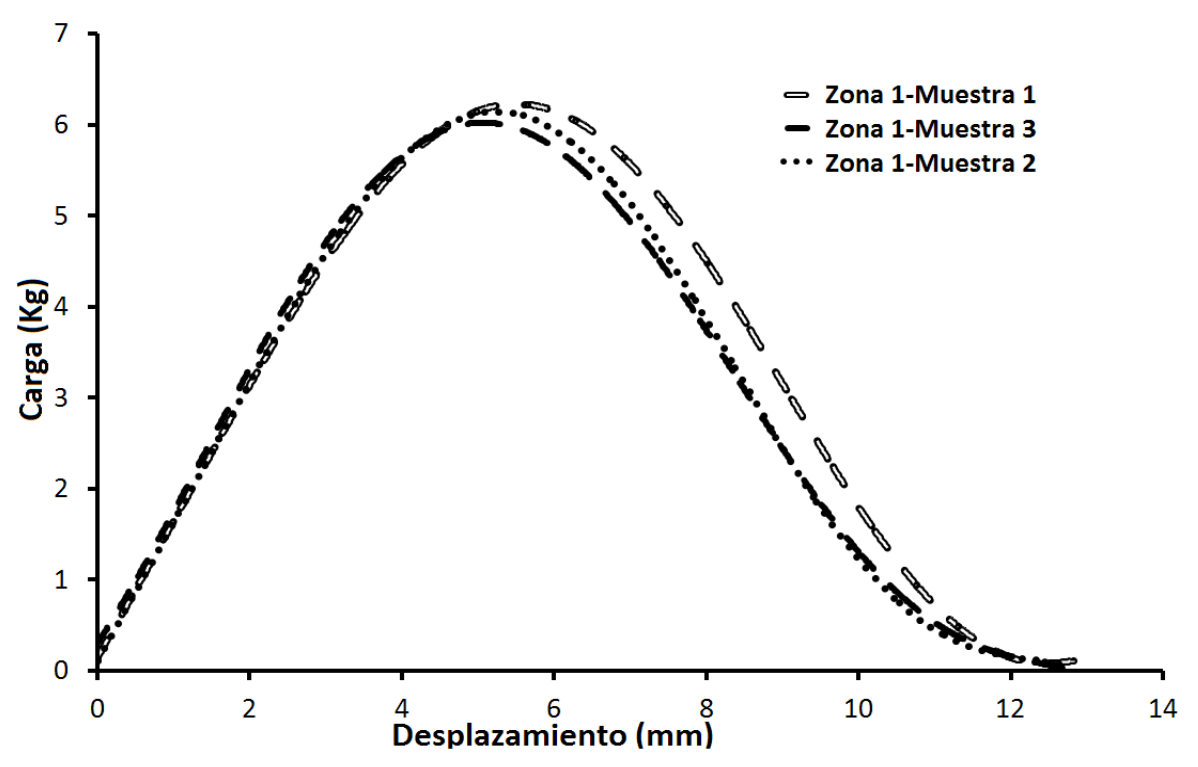

Figura 2. Resultados ensayo RTI en estado seco para Zona 1.

Se realizó el ensayo de Resistencia a Tracción Indirecta para la Zona 2 en estado seco, evidenciando antes y después de la carga máxima soportada un comportamiento similar en las muestras 4,5 y 6 , pero teniendo en cuenta que la muestra 6 presenta la carga máxima de más o menos $6,00 \mathrm{Kg}$, seguida de la muestra número 5 , mientras que la muestra 4 presenta menor resistencia con $5,50 \mathrm{Kg}$. En cuanto al desplazamiento de las tres muestras de esta zona, este resultó ser de $12,00 \mathrm{~mm}$; los resultados se muestran en la figura 3. 


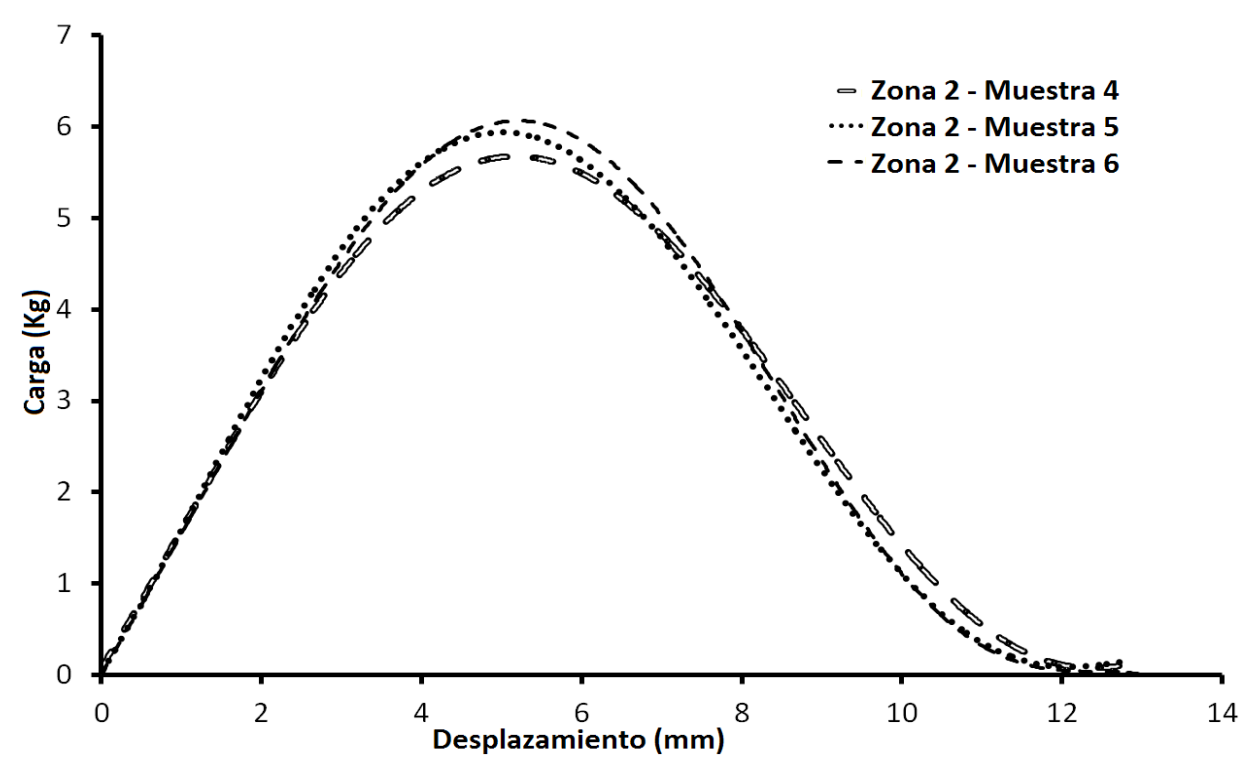

Figura 3. Resultados ensayo RTI en estado seco para Zona 2.

De las muestras ensayadas para la Zona 3, la 9 presentó mayor resistencia con $6,65 \mathrm{Kg}$, seguida de la muestra 8 con una resistencia de $6,58 \mathrm{Kg}$, mientras que la muestra 7 presentó una resistencia de $6,54 \mathrm{Kg}$; además, el comportamiento que presentan antes de llegar a la carga máxima es similar en la tendencia, pero después de esta cambia el comportamiento visualizándose en los desplazamientos, dado que la muestra 7 alcanza los 11,85 $\mathrm{mm}$, la muestra 8 llegó a desplazarse $12,27 \mathrm{~mm}$ y la muestra 9 llegó a un desplazamiento de 12,84 $\mathrm{mm}$, como se observa en la figura 4 .

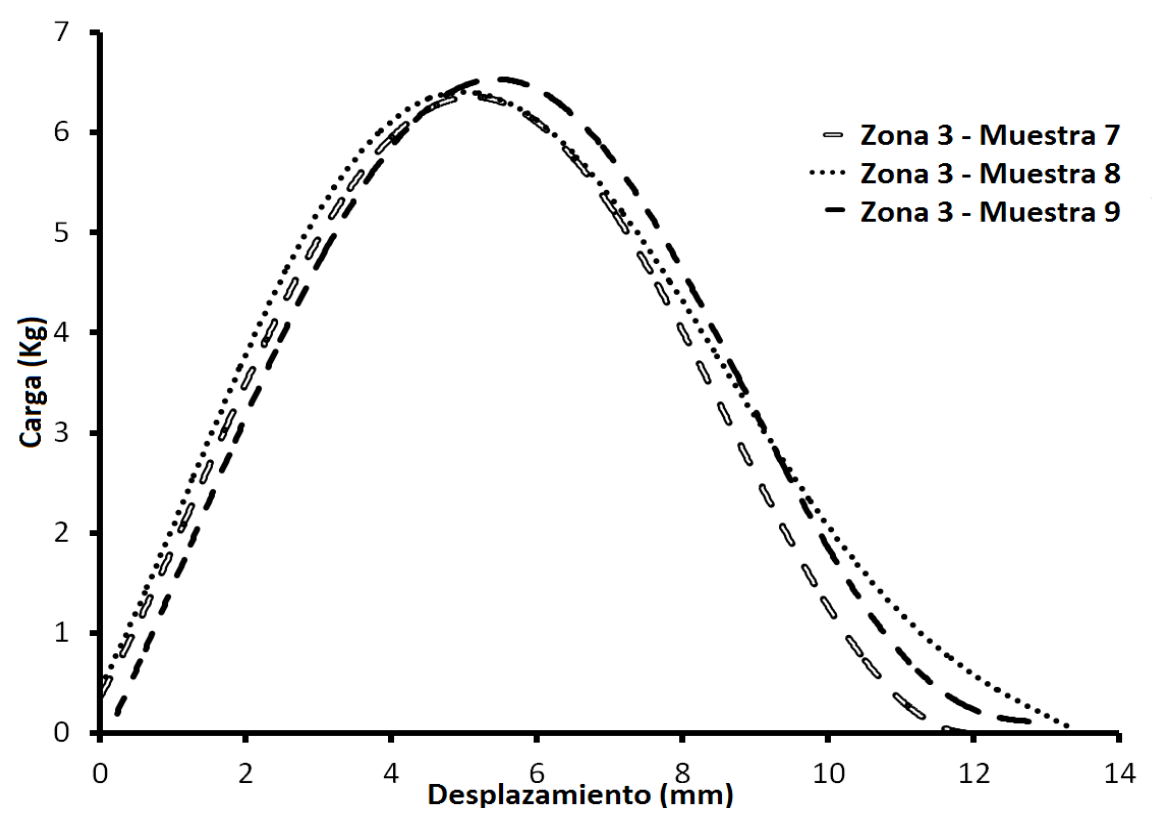

Figura 4. Resultados ensayo RTI en estado seco para Zona 3. 
Para la Zona 4, la muestra 12 presenta mayor resistencia llegando a $6,65 \mathrm{Kg}$, en seguida la muestra 10 llega a $6,58 \mathrm{Kg}$ y por último la muestra 11 llega a 6,54 Kg. Después de llegar a la carga máxima, el comportamiento funcional de las muestras difiere en el desplazamiento al cual llega, como se observa en la figura 5 .

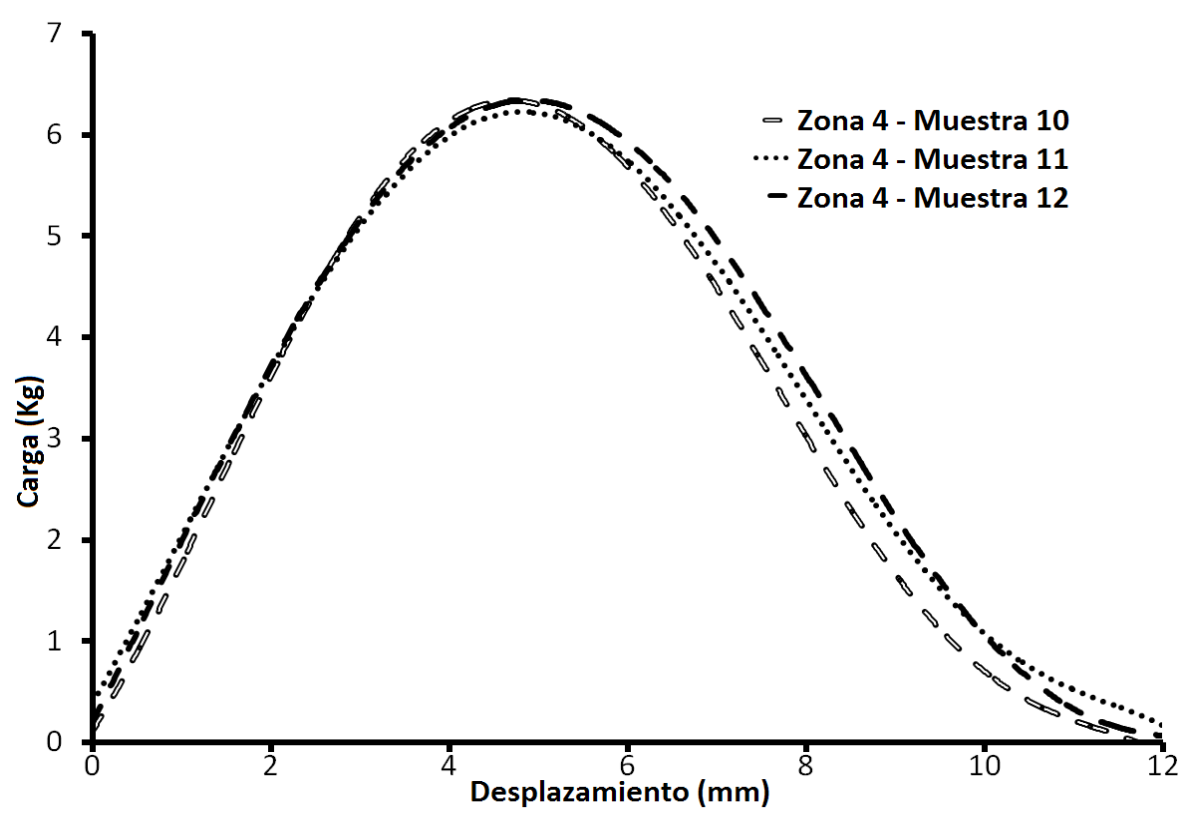

Figura 5. Resultados ensayo RTI en estado seco para Zona 4.

En las figuras 2 a 5 se presentan los resultados de las muestras falladas a Resistencia a Tracción Indirecta en estado seco, evidenciando que para las cuatro zonas, la carga máxima se presentó entre 6 y $7 \mathrm{Kg}$, pero las muestras de la Zona 3 tuvieron mayor resistencia y así mismo mayor desplazamiento.

A continuación se describen los resultados para las muestras que se ensayaron a Resistencia a Trac- ción Indirecta en estado húmedo. En la figura 6 se observa que la muestra 13 presenta la menor resistencia, siendo de 1,20 Kg; se evidencia mayor ductilidad que para las otras muestras llegando a un desplazamiento de $14,74 \mathrm{~mm}$. Por su parte, la muestra 14 presenta la mayor resistencia para la Zona 1 , con $2,61 \mathrm{Kg}$ y a su vez el menor desplazamiento, con 13,46 $\mathrm{mm}$. La muestra 15 presentó una resistencia de $2,56 \mathrm{Kg}$. 


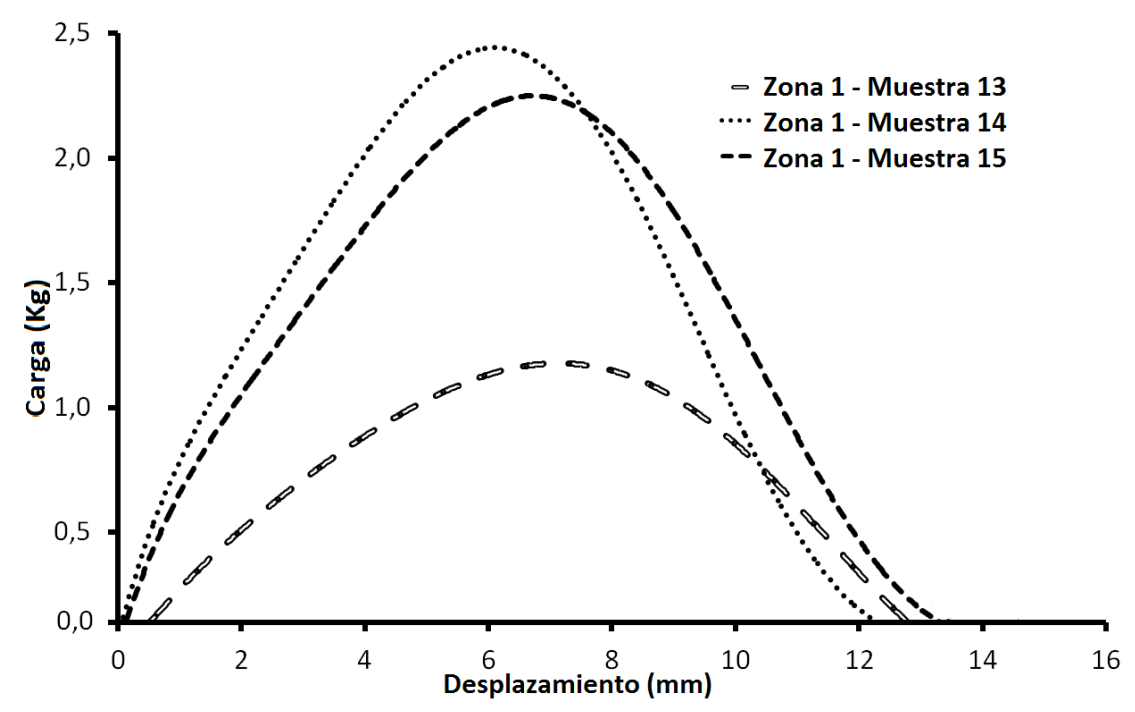

Figura 6. Resultados ensayo RTI en estado húmedo para Zona 1.

Los resultados arrojados para la Zona 2 varían tanto en la resistencia como en el desplazamiento. La muestra 16 presenta la mayor resistencia de la Zona con $1,70 \mathrm{Kg}$ y el menor desplazamiento de $13,10 \mathrm{~mm}$; en seguida la muestra 18 presenta una resistencia máxima de 1,40 Kg y un desplazamiento medio de $14,10 \mathrm{~mm}$. Por otra parte, la muestra 17 presenta la menor resistencia siendo de $1,20 \mathrm{Kg}$ y el mayor desplazamiento de $14,30 \mathrm{~mm}$, como se observa en la figura 7.

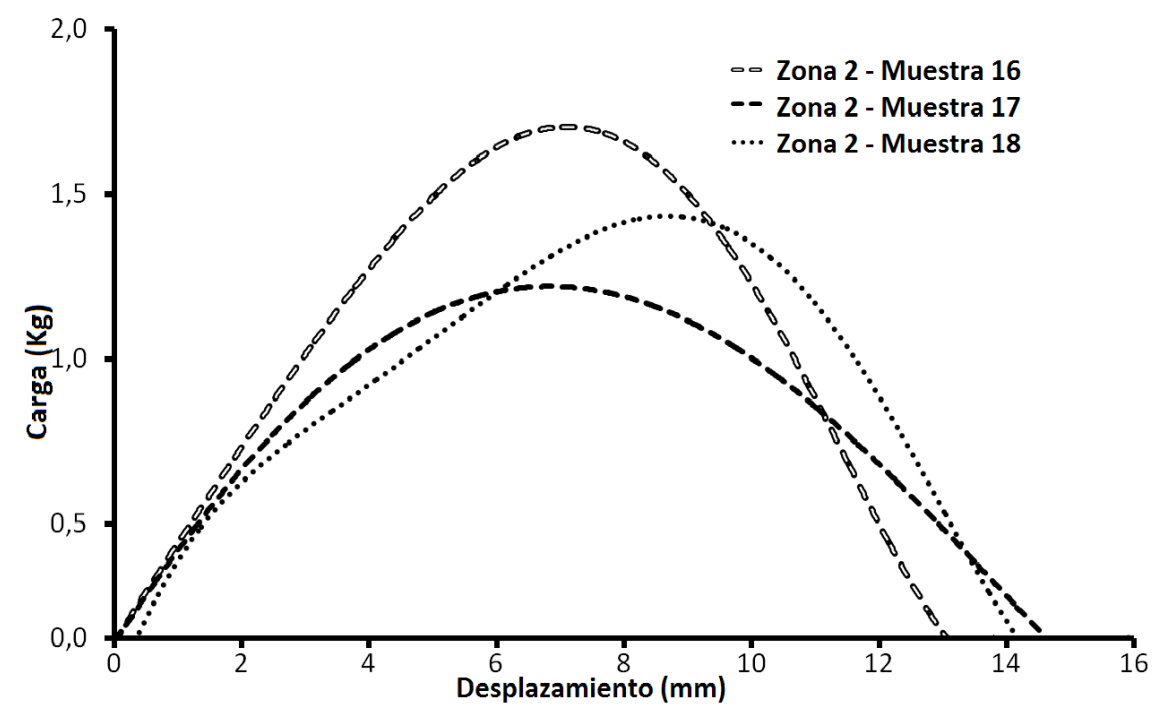

Figura 7. Resultados ensayo RTI en estado húmedo para Zona 2.

En la figura 8 se evidencia que la muestra 21 presenta la mayor resistencia siendo de $1,49 \mathrm{Kg}$, y un desplazamiento de $14,90 \mathrm{~mm}$; la muestra 19 presenta una resistencia de $1,30 \mathrm{Kg}$ en donde después de la carga máxima se denota una caída rápida en su comportamiento obteniendo el menor desplazamiento de la Zona, con $12 \mathrm{~mm}$. 


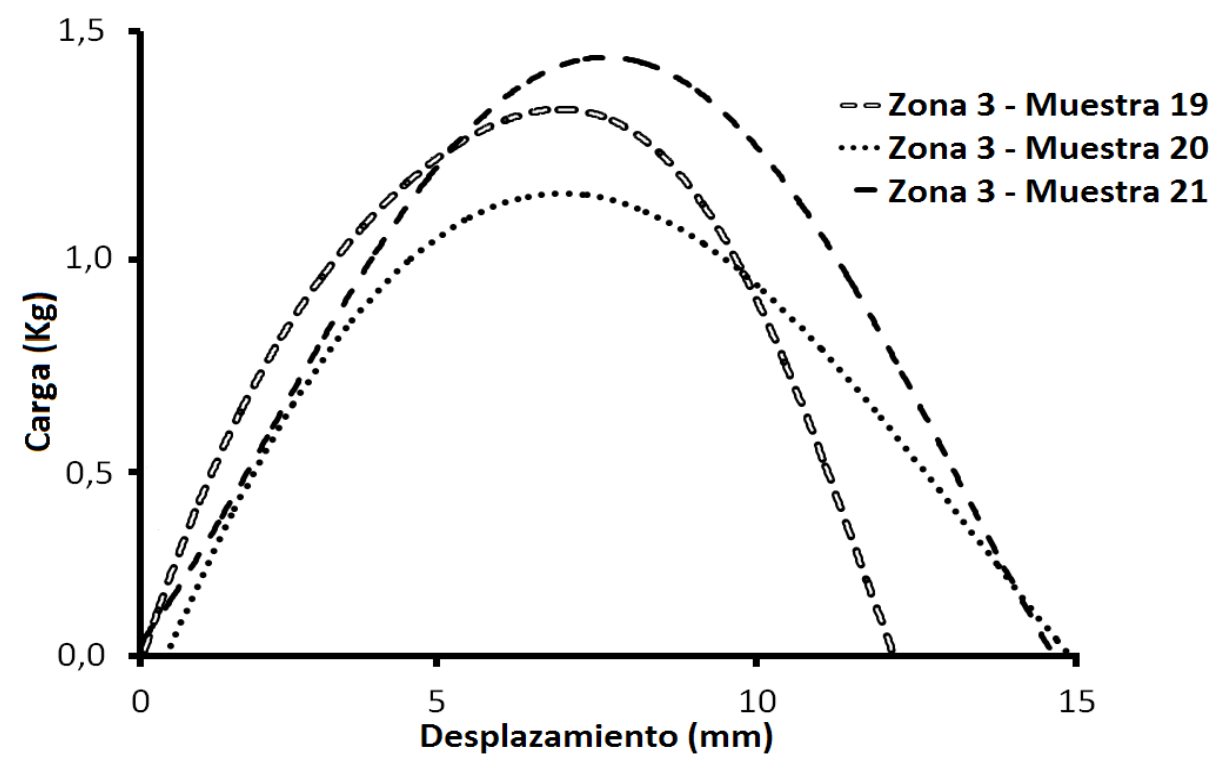

Figura 8. Resultados ensayo RTI en estado húmedo para Zona 3.

En la figura 9 se ilustran los resultados para la Zona 4 en estado húmedo, teniendo en cuenta que las muestras 23 y 24 presentan una resistencia máxima similar, pero después de esta el desplazamien- to es diferente. La muestra 22 alcanzó una carga máxima de $1,50 \mathrm{Kg}$ y el menor desplazamiento, siendo de $11,00 \mathrm{~mm}$.

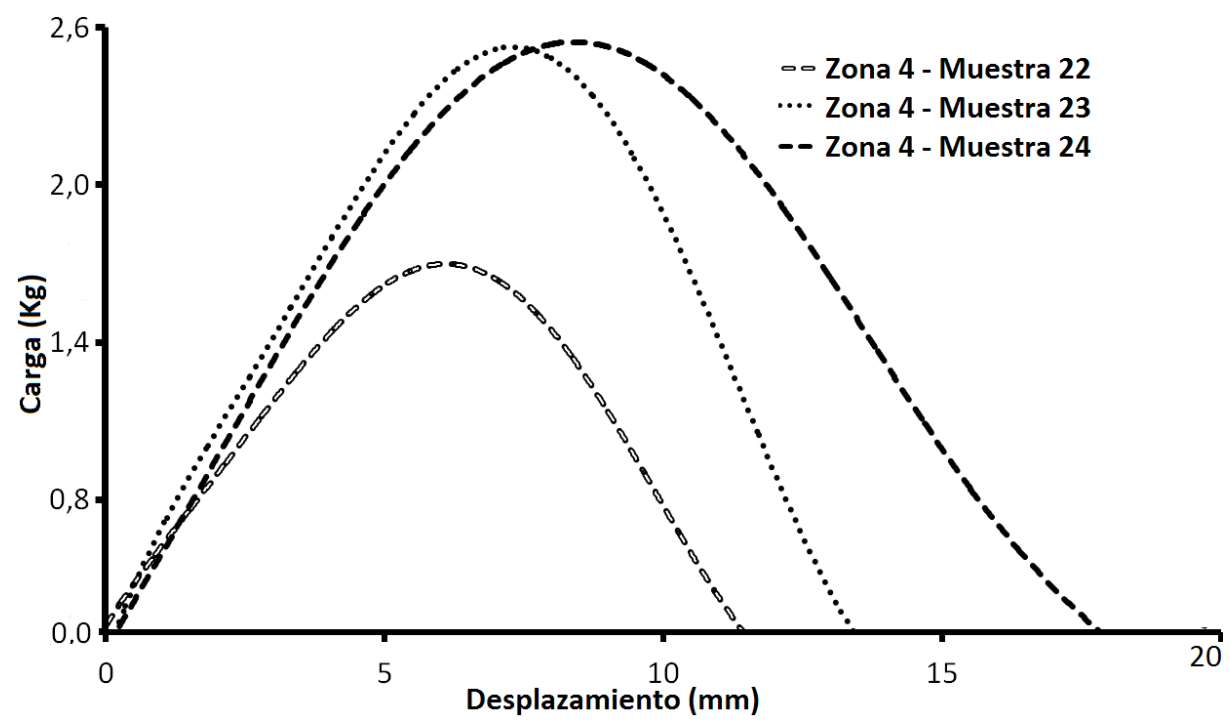

Figura 9. Resultados ensayo RTI en estado húmedo para Zona 4.

\section{Conclusiones}

Los resultados de Resistencia a Tracción Indirecta para las 4 zonas estudiadas, muestran que las pro- betas soportan cargas muy bajas con la metodología de mezclado a temperatura ambiente, para lo cual se recomienda hacer estudios adicionando material granular y un aditivo adicional, como 
emulsiones asfálticas. Resultados diferentes han sido reportados por Rondón y Reyes (2012), ya que en el proceso de trabajo del asfalto natural ellos aumentaron la temperatura para su uso.

El estudio de muestras de asfaltita en estado puro y con metodología de mezclado a temperatura ambiente, evidencian mayor ductilidad en las probetas en estado húmedo que en estado seco. Las muestras falladas en estado seco presentan mayor resistencia que las muestras falladas en estado húmedo, teniendo diferencias de $3,50 \mathrm{Kg}$.

Las tres muestras ensayadas, sometidas a un estado húmedo, exhiben para cada zona una diferencia notable en la carga máxima soportada y en la ductilidad presente. Caso contrario sucede en las muestras que se encontraban en estado seco, dado que presentan similitud en la resistencia máxima y en general en la representación gráfica que se visualiza.

\section{Agradecimientos}

Los autores agradecen a la Universidad Militar Nueva Granada, Colombia, por la financiación del proyecto DIS-1246.

\section{Referencias}

Barbosa, S. L. (2003). Ensayos de composición química, adherencia, compactación y resistencia. Universidad de Los Andes.

Berry, P. L., \& Reid D. (1993). Mecánica de Suelos. Vol. I. México D.F., México: Noriega Editores.
Instituto Nacional de Vías-INVÍAS. (1997). Especificaciones técnicas. Bogotá, Colombia: Editorial Escuela Colombiana de Ingeniería.

Osborn, J. Improved pavement material.U.S.,WO2002010285 A1. Febrero 07 de 2002. PCT/US2001/023515, julio 27 de 2000.

Rohde, L.; Pereira, J. A.; Pérez, W.; \& Vitorello, T. (2008). Using APT and laboratory testing to evaluate the performance of high modulus asphalt concrete for base courses in Brazil. En Proceedings of the 3rd International Conference on Accelerated Pavement Testing. Congreso llevada a cabo en Madrid, España.

Rondón, H. A., \& Reyes, F. A. (2012). Evaluación de las propiedades mecánicas de una mezcla densa en caliente modificada con asfaltita. Revista de Ingeniería, 36, 12-19. Recuperado de: http://www. redalyc.org/pdf/1210/121025826002.pdf

Ruíz, J., Reyes, O., \& Moreno A. (2013). Estudio del comportamiento de asfaltitas provenientes de $\mathrm{Ca}$ quetá en mezclas asfálticas de granulometría densa. En: XIX Simposio Colombiano sobre Ingeniería de Pavimentos. Simposio llevado a cabo en la Universidad Piloto de Colombia, Bogotá, Colombia.

Spielmann, P. E. (2000). Asphalt Roads. Londres, Reino Unido: Edward Arnold \& Co.

Vázquez, I. (2010). Ventajas y desventajas del uso de polímeros en asfaltos (Tesis de pregrado) Universidad Veracruzana, Veracruz, México. 